\title{
A new classification system for pebble and crystal shapes based on static equilibrium points
}

Tímea Szabó, Gábor Domokos

Department of Mechanics, Materials and Structures

Budapest University of Technology and Economics, Budapest

\begin{abstract}
The most widespread classification system for pebble shapes in geology is the Zingg system which relies on several length measurements. Here we propose a completely different classification system which involves counting static equilibria. We show that our system is practically applicable: simple hand experiments are suitable and easy to use to determine equilibrium classes. We also propose a simplified classification scheme called E-classification which is considerably faster in practice than the classical Zingg method. Based on statistical results of 1000 pebbles from several different geologic locations we show that E-classes are closely related to the geometric shape of pebbles. We compared E-classes to the Zingg classes, and we found that all the information contained in Zingg classification can be extracted from equilibrium classification. However, the new method is more sophisticated: it may help to identify shape attributes not discovered so far and it is able to store information on special geometries, e.g. on crystal shapes.
\end{abstract}

Key words: pebble, shape indices, Zingg classes, static equilibria, crystal

\section{Introduction}

The shape of sedimentary particles is an important physical property that facilitates facies differentiation, provides information about the history of the sediment and helps to characterize depositional environments (Graham and Midgley 2000). Analysis of the shape is also a key element in understanding bedload transport and sorting of sedimentary particles, since shape has an influence on the hydrodynamic behavior of particles in the transporting medium (Oakey et al. 2005). However, shape identification may also help in other fields,

Addresses: T. Szabó, G. Domokos: H-1111 Budapest, Múegyetem rkp. 3, K242, Hungary e-mails: tszabo@szt.bme.hu,domokos@iit.bme.hu

Received: May 28, 2010; accepted: October 10, 2010 
as particle shape is a complex function of lithology, particle size, the way and duration of transport, the energy of the transporting medium, the nature and extent of post-depositional weathering, and the history of sediment transport and deposition.

Although shape is a basic attribute of all objects, including pebbles, complete characterization of such three-dimensional shapes poses formidable difficulties. Sedimentologists have developed a number of standardized shape indices to roughly estimate and classify pebble shapes; these shape indices are derived from measurements of the length, breadth and thickness of a pebble. We will briefly review these classical pebble shape categories in the next section.

In this paper we propose a completely different classification scheme which does not rely on length measurements and shape indices; rather, it involves counting static equilibria. Static equilibria are points of the surface where the pebble is at rest when placed on a horizontal, frictionless support surface. While our previous study (Domokos et al. 2010) provided a theoretical basis for equilibrium classification, here we concentrate on applicability in field measurements as well as geologic verification.

We demonstrate that the new system is faster than traditional length measurements, that it is readily applicable in field work, and we give practical instructions for simple hand experiments. We also show how equilibrium classification can be naturally applied for crystal shapes; the new system is a clear indicator of crystal geometry.

One of our central objectives is to show that the number and type of static equilibria is closely related to the geometric shape of a pebble, i.e. the equilibrium class is a natural property which describes pebble geometry well. We present detailed statistical data of 1000 pebbles from different depositional environments, abrasion processes and pebble lithology. Different statistical results of different geologic locations suggest that the latter could be distinguished based on equilibrium classification, e.g. the new system is characteristic for a given geologic location. We compared the new equilibrium classification system to a well-known classical scheme (Zingg classes) and we found that equilibrium classification contains all the information contained in the classical method. However, the new system provides more detailed data and it may shed light on special shape features not discovered so far.

\section{Shape indices}

One of the important tasks of sedimentology is to sort the infinite number of conceivable particle shapes into a finite number of classes, because well-chosen classes can carry important information on the history of the sediment. Despite the extensive literature on the topic, there is little agreement on the best classification method for pebble shape analysis. A variety of shape indices and diagrammatical presentations of grain shape have been proposed in the past 
(Wentworth 1922; Zingg 1935; Krumbein 1941; Sneed and Folk 1958; Aschenbrenner 1956; Smalley 1967; Dobkins and Folk 1970). These methods for quantitative characterization of pebble shapes have been the subject of lively discussions recent years (e.g. Illenberger 1991, 1992a, 1992b; Benn and Ballantyne 1992; Woronow 1992; Graham and Midgley 2000; Oakey et al. 2005). These classification systems require the measurement of the three orthogonal axis lengths $\mathrm{L}>\mathrm{I}>\mathrm{S}$ of the approximating three-axial ellipsoid, where $\mathrm{L}$ is the longest, I the intermediate and S the shortest axis of the pebble. Based on these axis lengths a number of shape indices have been used, some of them to predict the hydraulic behavior of sediment grains. A thorough summary of shape indices is given by Blott and Pye (2008) and Illenberger (1991); see also Table 1.

Table 1

The most frequently used and most widespread shape indices

\begin{tabular}{|c|c|c|}
\hline Index & Formula & Author \\
\hline \multicolumn{3}{|l|}{ First-order indices } \\
\hline$\frac{S}{I}$ (flatness) & $\frac{S}{J}$ & Zingg (1935) \\
\hline $\begin{array}{l}I \\
I\end{array}$ & $\begin{array}{l}1 \\
I\end{array}$ & \\
\hline$\frac{T}{L}$ (elongation) & $\bar{L}$ & Zingg (1935) \\
\hline$\underline{S}$ (equancy) & $\underline{S}$ & Sneed \& Folk (1958) \\
\hline & $\bar{L}$ & Sheed \& Folk (1938) \\
\hline \multicolumn{3}{|l|}{ Second-order indices } \\
\hline$\underline{S^{2}}$ & $\underline{S^{2}}$ & \\
\hline$\overline{I L}$ & $\overline{I L}$ & \\
\hline$I^{2}$ & $I^{2}$ & \\
\hline$\overline{S L}$ & $\overline{S L}$ & \\
\hline$L^{2}$ & $L^{2}$ & \\
\hline$\overline{S I}$ & $\overline{S I}$ & \\
\hline \multicolumn{3}{|c|}{$\begin{array}{l}\text { Indices similar to second-order indices } \\
\end{array}$} \\
\hline Krumbein intercept sphericity & $\sqrt[3]{\frac{I S}{L^{2}}}$ & Krumbein (1941) \\
\hline Corey shape factor & $\frac{S}{\sqrt{L I}}$ & Corey (1949) \\
\hline Maximum projection sphericity & $\sqrt[3]{\frac{S^{2}}{L I}}$ & Sneed \& Folk (1958) \\
\hline Aschenbrenner shape factor & $\frac{L S}{I^{2}}$ & Aschenbrenner (1956) \\
\hline \multicolumn{3}{|l|}{ Other indices } \\
\hline Oblate-prolate index & $\frac{10\left(\frac{L-I}{L-S}-0.5\right)}{\frac{S}{L}}$ & Dobkins \& Folk (1970) \\
\hline Disc-rod index & $\frac{L-I}{L-S}$ & Sneed \& Folk (1958) \\
\hline Rod index & $\frac{S+I}{L}$ & Illenberger (1991) \\
\hline$\frac{L+S}{I}$ & $\frac{L+S}{I}$ & Illenberger (1991) \\
\hline Wentworth flatness index & $\frac{L+I}{2 S}$ & Wentworth (1922) \\
\hline
\end{tabular}


The first-order ratios of the three orthogonal axes are the easiest shape indices to conceptualize. Allowing reciprocal functions, second-order indices are products of two first-order indices. Most of the proposed shape indices can be related to these first- and second-order indices and two independent shape indices are required to produce a particle-form diagram. Most researchers use either the Zingg (1935) or the Sneed and Folk (1958) methods to graphically represent and classify pebble shapes. Zingg proposed a Cartesian coordinate system with $\mathrm{S} / \mathrm{I}$ and $\mathrm{I} / \mathrm{L}$ as indices for the shape diagram, where $\mathrm{S} / \mathrm{I}$ is a measure of flatness and I/L is a measure of elongation (Fig. 1a).

a

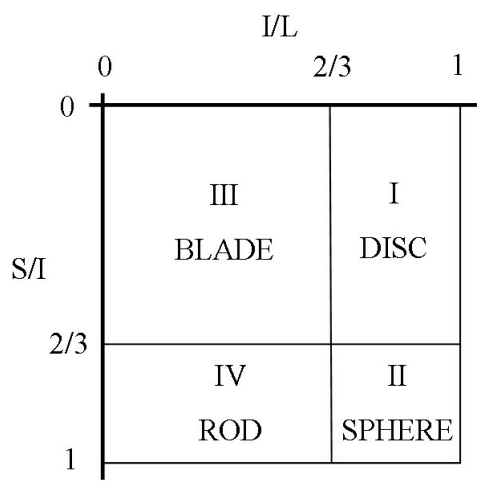

b

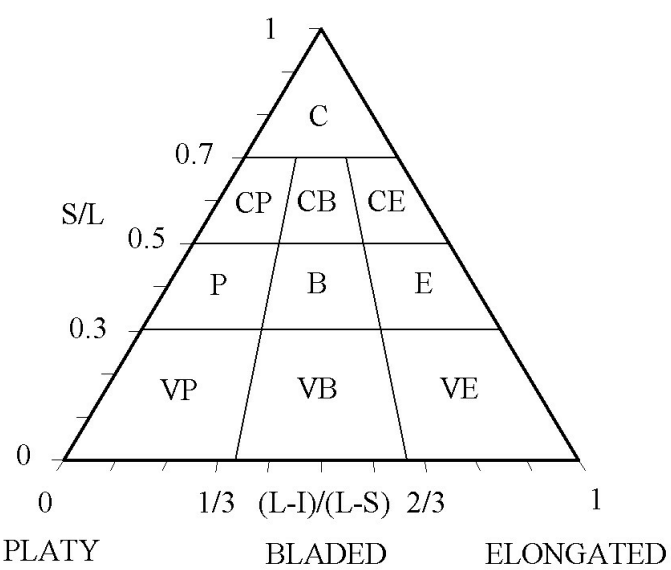

Fig. 1

a) Zingg diagram with 4 classes: Disc (I), Sphere (II), Blade (III), Rod (IV)

b) Triangular shape classification diagram by Sneed and Folk; the classes are: Compact (C), Compactplaty (CP), Compact-bladed (CB), Compact-elongated (CE), Platy (P), Bladed (B), Elongated (E), Very platy (VP), Very bladed (VB), Very elongated (VE)

The Zingg diagram is a simple and clear classification system; however, Sneed and Folk (1958) concluded that the Zingg classification is an inadequate tool, as it only contains four classes. They suggested that pebble shapes should be plotted on a triangular diagram, where $\mathrm{S} / \mathrm{L}$ is plotted against the Disc-rod index (Fig. $1 \mathrm{~b}$, see also Table 1), and they divided the diagram into 10 shape classes. Several authors have argued whether the Zingg or the Sneed and Folk system is the most suitable classification scheme; Blott and Pye (2008) concluded that the Zingg diagram provides a more even distribution of the shape continuum than the triangular Folk diagram. Zingg classification is probably the easiest and most logical way to estimate pebble shapes; therefore in this paper we will use Zingg classes as a basis for comparison to our new classification system. However, connection with other classification methods could also be demonstrated. 
The above-mentioned classical pebble categories rely on length measurements, which inevitably cause inaccuracies in the classification method because it is often uncertain as how to L, I and S should be defined and also because measurements involve a degree of error and they might be quite tedious. Most researchers have agreed that $\mathrm{L}, \mathrm{I}$ and $\mathrm{S}$ should be perpendicular to each other, but they do not need to intersect at a common point. However, as shown by Blott and Pye (2008), the exact directions of the three axes are uncertain even in the case of a simple cube. If we define the $\mathrm{L}$ dimension as the maximum 'caliper' dimension (as most authors do), then the longest dimension is the body diagonal of the cube and the two axes perpendicular to this are not of equal length. Blott and Pye proposes that the L, I and S dimensions should be defined as the side lengths of the smallest imaginary box which can contain the particle. This definition leads to an accurate calculation in the case of cube; the three axis lengths are equal to each other (and to the side lengths of the cube). However, this method is rather complicated to achieve: the examiner must orientate the particle by eye so that $S$ and I prescribe the smallest projected area.

It is also apparent that classical pebble-shape categories involve arbitrarily chosen constants to separate shape classes from each other. The Zingg system uses an axis ratio value of $2 / 3$ to discriminate the four classes. The classical Zingg system can be unified if an internal parameter $0 \leq p \leq 1$ is introduced. In the classical Zingg system of $p=2 / 3$, the generalized Zingg classes can be given as

$$
\begin{array}{lll}
Z_{\mathrm{p}}(\mathrm{I}): & \text { Disc } & \mathrm{I} / \mathrm{L} \geq \mathrm{p} \text { and } \mathrm{S} / \mathrm{I} \leq \mathrm{p} \\
\mathrm{Z}_{\mathrm{p}}(\mathrm{II}): & \text { Sphere } & \mathrm{I} / \mathrm{L} \geq \mathrm{p} \text { and } \mathrm{S} / \mathrm{I} \geq \mathrm{p} \\
\mathrm{Z}_{\mathrm{p}}(\mathrm{III}): & \text { Blade } & \mathrm{I} / \mathrm{L} \leq \mathrm{p} \text { and } \mathrm{S} / \mathrm{I} \leq \mathrm{p} \\
\mathrm{Z}_{\mathrm{p}}(\mathrm{IV}): & \text { Rod } & \mathrm{I} / \mathrm{L} \leq \mathrm{p} \text { and } \mathrm{S} / \mathrm{I} \geq \mathrm{p}
\end{array}
$$

Although this not was explicitly defined, Zingg assumed that the $p=2 / 3$ value is universally optimal; however, later we will show that the optimal choice of $p$ depends on the investigated sample. The value of $\mathrm{p}$ has a great influence on the result of the classification, i.e. classification schemes derived from length measurements are also ambiguous in that sense.

In this paper we propose an alternative classification system which does not suffer from these ambiguities (while inevitably, it includes others!). Our approach is very speedy and does not contain any arbitrarily introduced constants or directions. Our system relies on the (integer) number and type of static equilibria which we outline below.

\section{Equilibrium classes}

In this section we summarize in a nutshell the results of previous studies, focusing on the practical side of equilibrium classification system. For a detailed mathematical background, see Várkonyi and Domokos (2006); also Domokos et al. (2010). 
Static equilibria are points of the surface where the pebble is at rest on a horizontal, frictionless support. When placed on a horizontal surface, a homogeneous, convex, rigid body will come to rest at a stable point of equilibrium and it will return there after a small perturbation. For example, a cube will have 6 stable equilibria on the 6 faces. Unstable points of equilibria appear still as balance points when placed on a horizontal surface; however, the body will not return there after small perturbations. There are two generic versions of unstable equilibrium points: unstable maxima and saddles. For example, the cube has 8 unstable points (maxima) on the 8 vertices and 12 saddle type equilibria on the 12 edges.

A typical convex body may have three types of equilibria: stable, unstable and saddle type equilibria and our approach relies on counting these equilibrium points. If equilibrium points are well isolated, i.e. they are sufficiently apart, they can be easily identified by hand experiments. In such experiments small perturbations, realized by slightly tossing the object in one direction or the other, play a key role. Typical points of a surface are non-equilibrium points. When placed on such a point on a horizontal surface, the body will roll away, always in
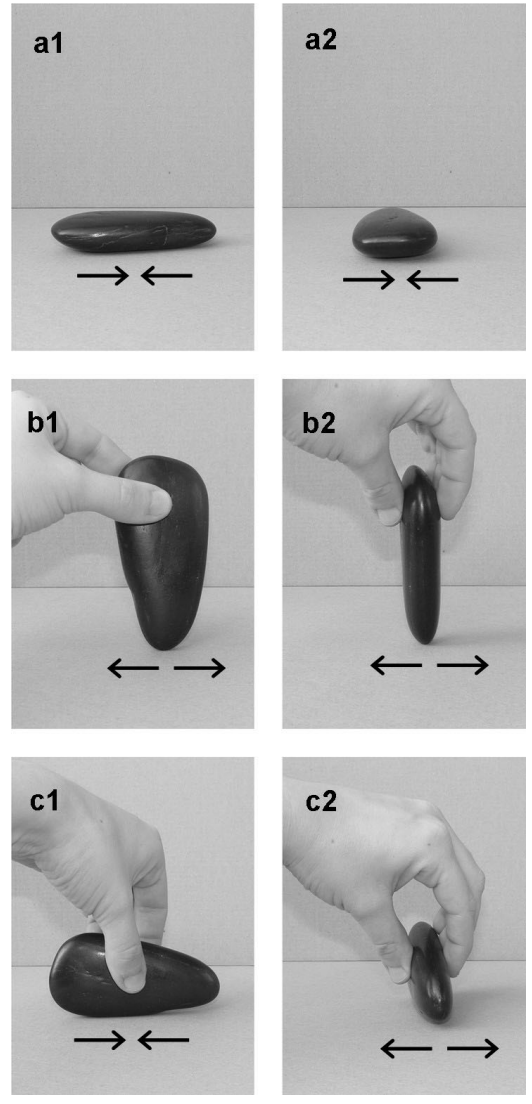

the same direction, even if we toss it slightly in a different way. Stable points are most easily identified in a hand experiment, as stable points behave as attractors (Fig. 2a), i.e. the body will always roll back to a stable point from a nearby location, no matter in which direction it is tossed away. Unstable points act as repellors, i.e. the body will always roll away from an unstable point;

Fig. 2

Counting equilibrium points of a flat pebble in a hand experiment. Arrows show if pebble rolls away from the equilibrium point or rolls back to the equilibrium point if we toss it slightly away in that direction.

a) when placed on a horizontal surface, the pebble will come to rest at a stable point of equilibrium and it will return there after small perturbation

b) constrained in the principal plane, vertical position, one of the maxima along the edge still appears as unstable equilibrium: pebble is stable laterally (constrained by hand, see b2 side view); however, unstable in the longitudinal direction (b1 frontal view) c) constrained in the principal plane, vertical position, one of the pebble's saddle points along the edge appears as a stable equilibrium: pebble is stable both laterally (constrained by hand, $\mathrm{c} 2$ side view) and longitudinally (c1 frontal view) 
however, unlike the case of non-equilibrium points, the direction of the roll is ambiguous; it depends entirely on the direction in which it is tossed. Differentiation between unstable points and saddle points requires more attention from the examiner. In hand experiments saddle points behave similarly to repellors; nevertheless, in the case of flat, disc-like objects they can be much more easily identified. In such cases saddles and unstable maxima are placed along the large perimeter of the flat object. By constraining the object vertically in the plane of the large perimeter, as the object rolls around the perimeter, saddles appear as attractors in this constrained problem, while unstable points remain repellors (Fig. $2 b, c)$. Since most pebbles tend to be flat, this is probably the easiest way to count unstable and saddle-type equilibria.

Static equilibrium points can be counted in such simple hand experiments. Let us denote the number of stable equilibrium points by $S$, the number of unstable equilibria by $U$ and the number of saddle type equilibria by $\mathrm{H}$. The Poincaré-Hopf Theorem (Arnold 1998) establishes the relationship S+U-H=2. Based on this result, we can define a unique classification for convex, rigid bodies based on the number and type of their equilibria (Várkonyi and Domokos 2006): class $\{S, U\}$ contains all convex, rigid bodies with $S$ stable and $U$ unstable equilibria. Thus, only two types of equilibria need to be counted in a hand experiment; the third type can be either computed or used to verify the experiment. For example, a cube is in class $\{6,8\}$ with $S=6$ stable and $U=8$ unstable equilibria. Although in Várkonyi and Domokos (2006) it was shown that all classes are non-empty, most classes are not easy to visualize. Figure 3 illustrates some equilibrium classes by characteristic shapes. (The illustrations do not represent all characteristic shapes of the given class.) The Gömböc (Várkonyi and Domokos 2006) is in class $\{1,1\}$; a characteristic example for class $\{2,2\}$ is a tri-axial ellipsoid. Observe that polyhedral shapes appear in the lower right corner of the table in the shaded wedge-shaped segment. Probably the most obvious examples for polyhedral shapes in geology are crystals. This indicates that equilibrium classification is not only a natural tool for classifying pebbles (as we later show), but it also can be applied to describe crystal shapes.

\section{Crystal shapes}

Crystal shapes are traditionally classified into the following 7 crystal systems: the triclinic, monoclinic, orthorombic, tetragonal, trigonal, hexagonal and the cubic system. Although there is a detailed and abundant literature on crystal shapes and various methods - including the Zingg method (Mock and Jerram 2005) - are used to describe the geometry and symmetry of crystals (Hahn 2002), equilibrium classification may provide new information on these special shapes.

Although equilibrium classification method based on hand experiments can only work on a relatively large single crystal, in fact in most cases the shape of a single crystal is so characteristic that we do not even need to take the crystal into 


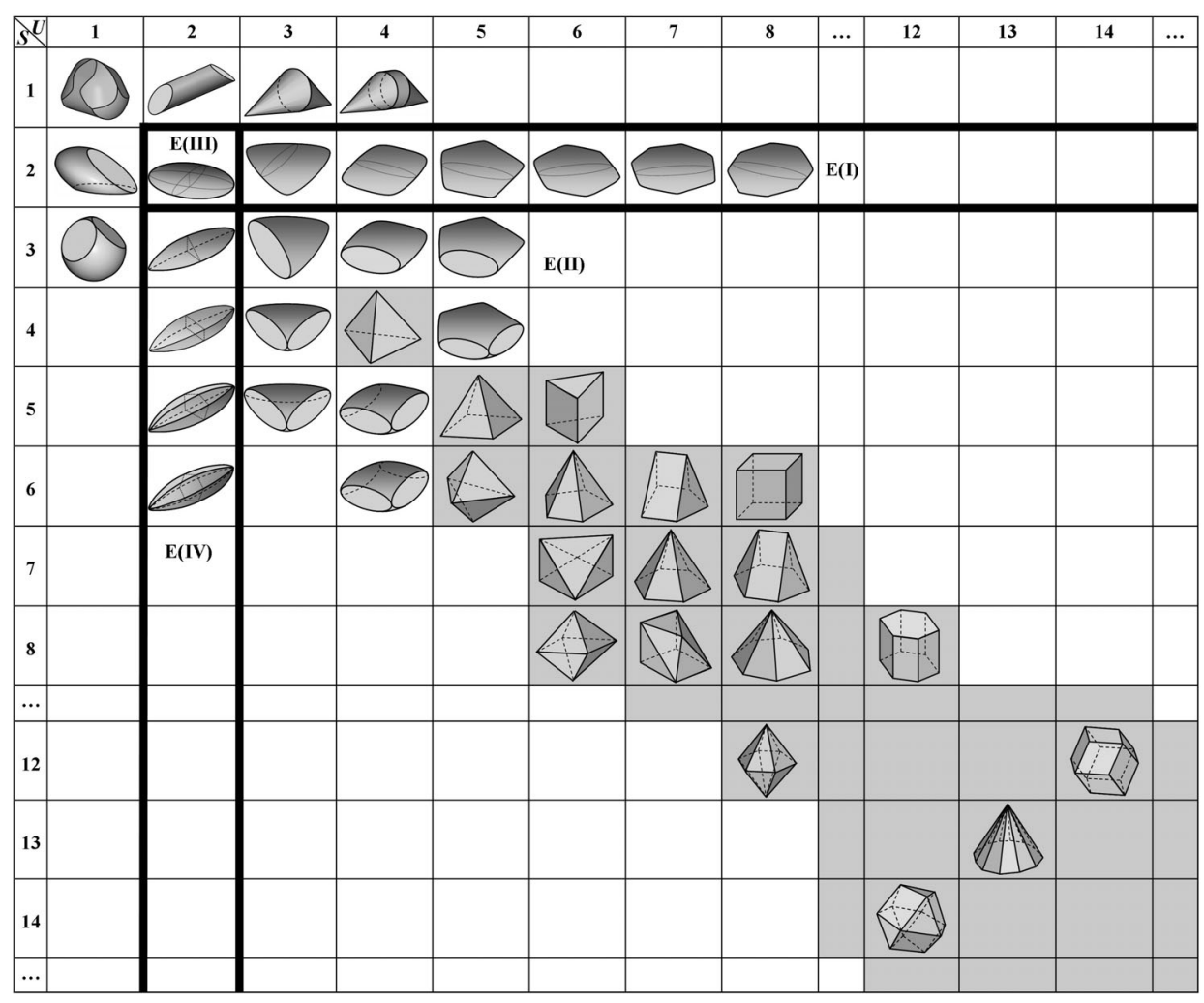

Fig. 3

Equilibrium classes for 3D bodies: examples for some characteristic shapes. Rows (S) denote number of stable equilibria, columns (U) denote number of unstable equilibria. Várkonyi and Domokos (2006) established the existence of $\{1,1\}$ and created an object belonging to this class: it is called the Gömböc. The tri-axial ellipsoid (which is used to approximate pebble shape in the Zingg system) is in $\{2,2\}$. Observe that shapes in row 2 (class E(I)) are rather flat whereas shapes in column 2 (class E(IV)) are rather thin. Also, observe that polyhedral (crystal) shapes emerge toward the lower right corner of the table (shaded wedge-shaped segment). Some typical crystal shapes in the table: pyramids with nsided base, $\mathrm{S}=\mathrm{U}=\mathrm{n}+1$ : tetrahedron $\{4,4\}$, tetragonal pyramid $\{5,5\}$, hexagonal pyramid $\{7,7\}$, dihexagonal pyramid $\{13,13\}$; dipyramids with $n$-sided base, $S=2 n, U=n+2$ : trigonal dipyramid $\{6,5\}$, octahedron $\{8,6\}$, hexagonal dipyramid $\{12,8\}$; n-sided prisms with $S=n+2, U=2 n$ : trigonal prism $\{5,6\}$, cube $\{6,8\}$, hexagonal prism $\{8,12\}$; other crystal shapes: cuboctahedron $\{14,12\}$, rhombic dodecahedron $\{12,14\}$

our hand, the equilibrium class can be determined just by visual inspection. The shape of a single crystal is usually a minimal polyhedron, i.e. each face of the polyhedron contains a stable equilibrium point, each vertex is an unstable equilibrium point and each edge contains a saddle type equilibrium. Let us denote the number of faces, vertices and edges by F, V and E, respectively. For minimal polyhedra Euler's formula $\mathrm{F}+\mathrm{V}-\mathrm{E}=2$ is equivalent to the Poincare-Hopf 
relationship $\mathrm{S}+\mathrm{U}-\mathrm{H}=2$ because $\mathrm{S}=\mathrm{F}, \mathrm{U}=\mathrm{V}$ and $\mathrm{H}=\mathrm{E}$. Therefore, to identify $\mathrm{E}-$ classes, the only task is to count the faces $(\mathrm{F})$ and the vertices $(\mathrm{V})$ of the polyhedron, determining the equilibrium class $\{\mathrm{F}, \mathrm{V}\}$ of the crystal. Minimal polyhedra are positioned in the shaded wedge-shaped segment of the table in Fig. 3, i.e. outside the wedge no minimal polyhedra exist. This statement can be proven along the following line: as each face is bounded by at least three edges, we have $3 \mathrm{~F} \leq 2 \mathrm{E}$. From Euler's formula $(\mathrm{F}+\mathrm{V}-\mathrm{E}=2)$ we have $2 \mathrm{E}=2 \mathrm{~F}+2 \mathrm{~V}-4$. The previous two imply $3 \mathrm{~F} \leq 2 \mathrm{~F}+2 \mathrm{~V}-4$ from which $\mathrm{F} \leq 2 \mathrm{~V}-4$ and, finally, $\mathrm{S} \leq 2 \mathrm{U}-4$ follows. Similarly, at least three edges start at each vertex, so we have $3 \mathrm{~V} \leq 2 \mathrm{E}$ which leads to $\mathrm{U} \leq 2 \mathrm{~S}-4$. Relationships $\mathrm{S} \leq 2 \mathrm{U}-4$ and $\mathrm{U} \leq 2 \mathrm{~S}-4$ determine the shaded wedge-shaped segment of the S-U table.

Table 2 illustrates equilibrium classification of crystals with some examples. Equilibrium class is very easy to determine; nevertheless it is a clear indicator of crystal geometry, although it uses only two integer numbers.

\section{Table 2}

Examples for equilibrium classes of single crystals. Crystal shapes are minimal polyhedra which are positioned in the shaded wedge-shaped segment of the table in Figure 3

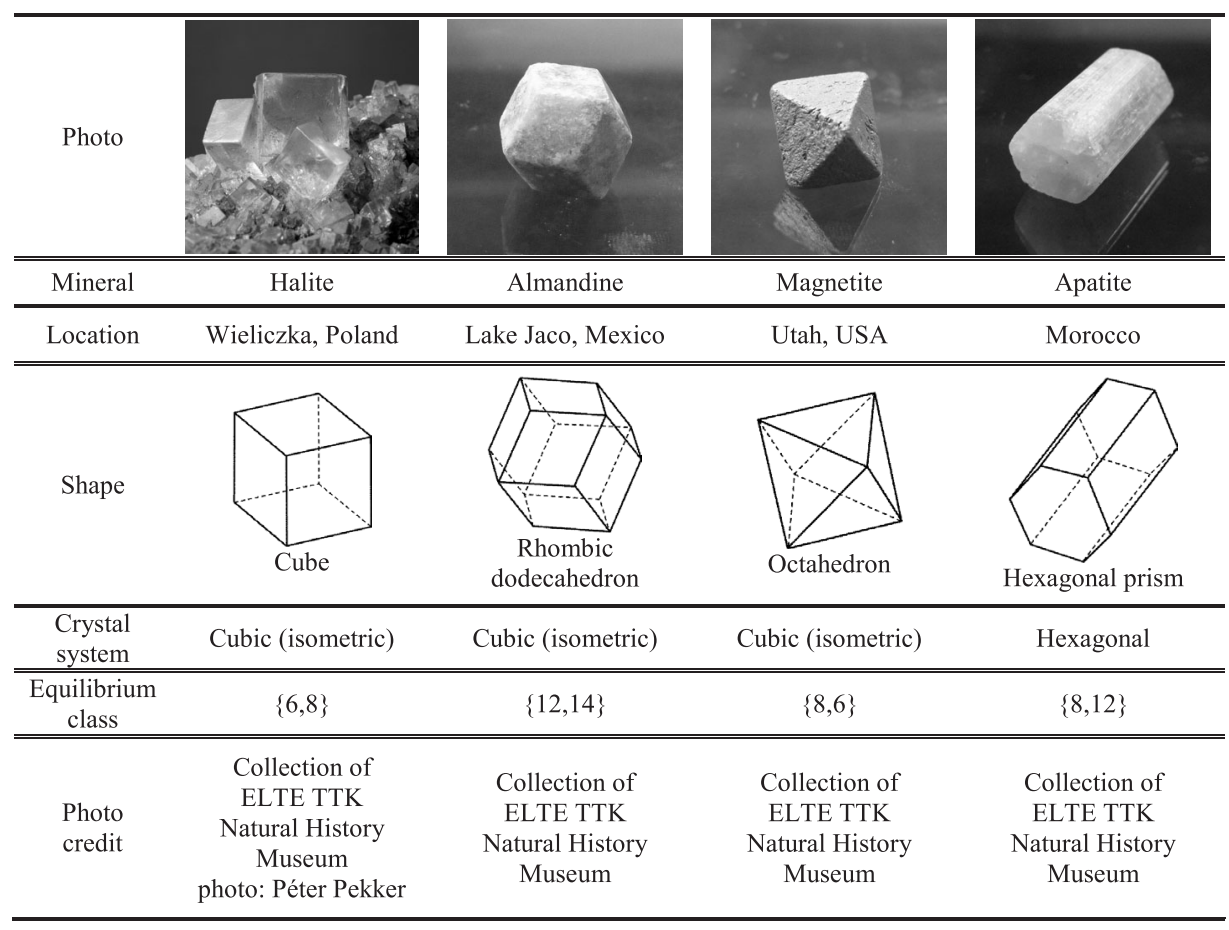




\section{E-classification and geometric shapes}

Equilibrium classification is not only an adequate tool for classifying crystal shapes; it also describes the shape of pebbles well, as we will show it in this section. Our main goal is to show how shape information can be extracted from the new equilibrium classification system and to demonstrate the connection between equilibrium classification and the classical Zingg system.

For easier comparison with the Zingg system we introduce the simplified Eclassification. The latter is based on equilibrium classes; however, it is radically simplified (see Fig. 3) as follows:

$\begin{array}{ll}\text { E(I): } & \text { E-classes }\{2, \mathrm{U}\}, \mathrm{U}>2 \\ \text { E(II): } & \text { E-classes }\{\mathrm{S}, \mathrm{U}\}, \mathrm{S}, \mathrm{U}>2 \\ \text { E(III): } & \text { E-class }\{2,2\} \\ \text { E(IV): } & \text { E-classes }\{\mathrm{S}, 2\}, \mathrm{S}>2\end{array}$

Pebbles with S,U=1 are extremely rare (Várkonyi and Domokos 2006), so these classes do not appear in the above simplified scheme. E-classification has the additional advantage that it is very easy to apply in hand experiments: the examiner has only to decide whether the pebble has two or more than two (stable and unstable) equilibrium points. This simplified scheme is a very speedy process; in the case of most pebbles it can be done in seconds.

Our goal is to show how E-class is related to the geometric shape of the pebble. Although E-class does not exactly determine the geometric shape, in some cases the shape of the pebble is so characteristic that the E-class can be determined

\begin{tabular}{|c|c|c|}
\hline $\begin{array}{c}\text { E-CLASSES } \\
\left(Z_{p} \text {-classes }\right) \\
\end{array}$ & $\begin{array}{c}\mathbf{U}=\mathbf{2} \\
(I / L<p)\end{array}$ & $\begin{array}{c}\mathbf{U}>\mathbf{2} \\
(I / L>p)\end{array}$ \\
\hline $\begin{array}{c}\mathbf{S}=\mathbf{2} \\
(S / I<p)\end{array}$ & $\begin{array}{c}\mathbf{E ( I I I )} \\
\left(Z_{p}(\text { III):Blade })\right.\end{array}$ & $\begin{array}{c}\mathbf{E}(\mathbf{I}) \\
\left(Z_{p}(I): D i s c\right)\end{array}$ \\
\hline $\begin{array}{c}\mathbf{S}>\mathbf{2} \\
(S / I>p)\end{array}$ & $\begin{array}{c}\mathbf{E}(\mathbf{I V}) \\
\left(Z_{p}(I V): R o d\right)\end{array}$ & $\begin{array}{c}\mathbf{E}(\mathbf{I I}) \\
\left(Z_{p}(I I): \text { Sphere }\right)\end{array}$ \\
\hline
\end{tabular}

Fig. 4

Four characteristic examples for E-classes. Typically, flat pebbles belong to $E(I)$, elongate pebbles belong to $\mathrm{E}(\mathrm{IV})$, flat and elongate pebbles belong to E(III). Pebbles neither flat nor elongate belong to $\mathrm{E}(\mathrm{II})$. E-classes $\mathrm{E}(\mathrm{i})$ are closely related to the generalized Zingg classes $Z_{p}(i)$. $\mathrm{Z}_{\mathrm{p}}$-classes of the 4 characteristic pebbles are shown in brackets (cf. Fig. 1a) even without counting, just by brief visual inspection. Figure 4 illustrates Eclasses with 4 characteristic pebbles: flat objects will typically belong to the simplified E-class E(I); elongated objects will typically belong to the simplified E-class E(IV); flat and elongated objects belong to $\mathrm{E}(\mathrm{III})$. Objects neither flat nor elongated typically belong to E(II) (cf. Fig. 3). This indicates that simplified E-classes E(i) are closely related to the generalized Zingg classes $Z_{p}(i)$ as the axis ratio $S / I$ is a measure of flatness and $\mathrm{I} / \mathrm{L}$ is a measure of elongation.

As mentioned before, in the case of E-classification the examiner must make two binary choices by determining whether the number of stable points (S) and the number of 
unstable points $(\mathrm{U})$ is greater or equal to 2 . Whether $\mathrm{S}$ is greater or equal to 2 can be often be decided without even taking the pebble in hand; as pointed out above, flat pebbles typically have $S=2$. Similarly, very elongated pebbles typically have $U=2$. If a pebble is neither very elongated nor very flat then the examiner must begin counting $S$ and $U$. If any of these numbers exceed 2, counting may cease: from the viewpoint of E-classes no further information is required. However, if we are interested in the exact number of stable and unstable equilibria, it sometimes helps - especially in case of complicated shapes - to mark the identified equilibrium points on the surface (thus avoiding counting them twice).

Figure 4 suggest that simplified E-classes E(i) are closely related to the generalized Zingg classes $Z_{p}(i)$. We will validate this statement with quantitative, statistical data in the next section.

\section{Statistical comparison of E-classes and Z-classes}

We examined the agreement between E-classes and $Z_{p}$-classes on 20 collected pebble samples (each of them consisting of 50 pebbles). The samples are from

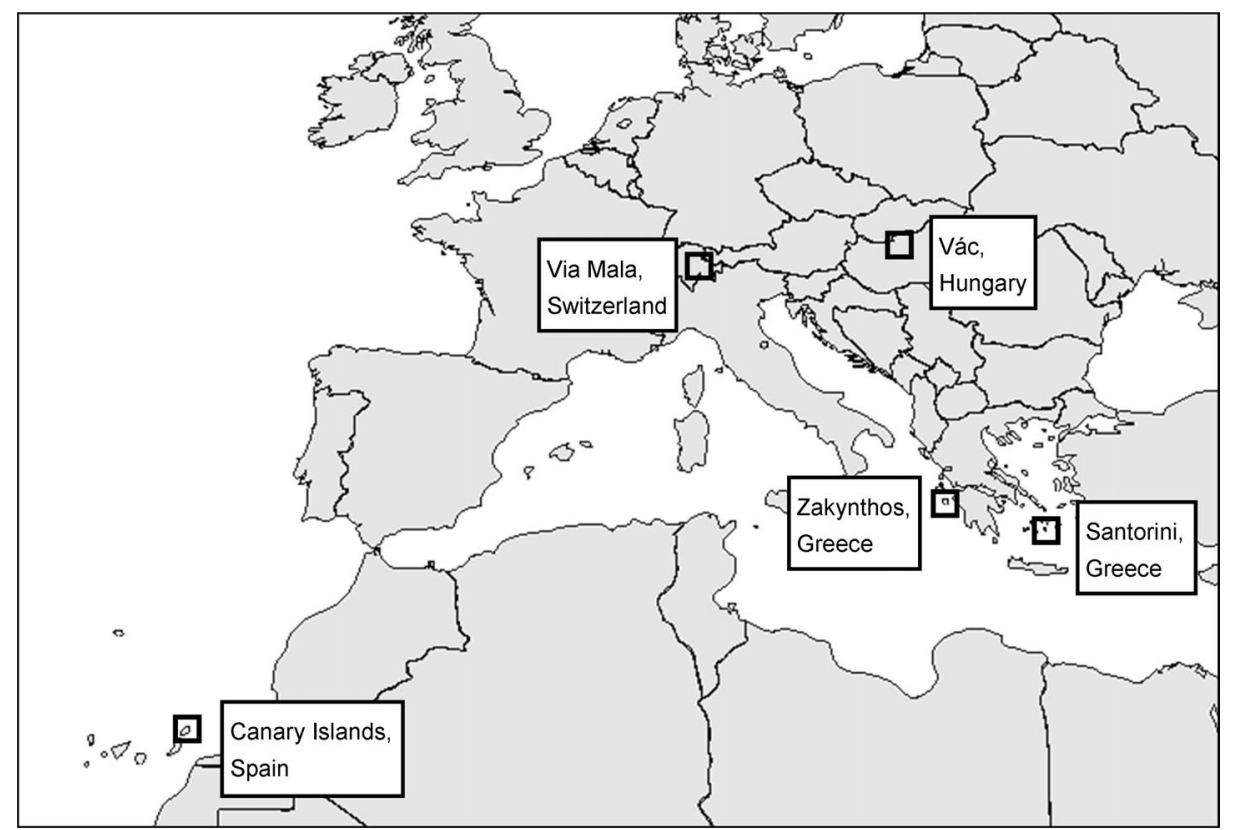

Fig. 5

Place of origin of the 20 pebble samples. Each sample consists of 50 pebbles. Geologic locations are rather different: abrasion processes (pebbles on the seashore and river-bank) and rock types differ from each other (see also Table 4) 
several different geologic locations (Fig. 5), representing different depositional environments, abrasion processes and pebble lithology.

We determined the E-class of all pebbles as well as their axis ratios $\mathrm{S} / \mathrm{I}$ and $\mathrm{I} / \mathrm{L}$. Based on the latter, the $\mathrm{Z}_{\mathrm{p}}$-class can be determined if the parameter $\mathrm{p}$ is given (in

Table 3

A simple table for statistical comparison between E-classes and $Z_{p}$-classes (Sample data refer to the case $\mathrm{p}=0.84$ ). If time allows it is worth using the "full" counting process, and record the exact number of $\mathrm{S}$ and $\mathrm{U}$. If time plays an important role, from the point of E-classification it is enough to record whether $\mathrm{S}$ (or $\mathrm{U}$ ) is greater or equal to 2

\begin{tabular}{|c|c|c|c|c|c|c|c|c|c|c|}
\hline \multirow[b]{2}{*}{$\begin{array}{c}\text { ID } \\
\text { number }\end{array}$} & \multicolumn{3}{|c|}{ Axes (mm) } & \multicolumn{2}{|c|}{ Equilibria } & \multicolumn{2}{|c|}{ Axis ratios } & \multirow{2}{*}{ E-class } & \multirow{2}{*}{$\mathrm{Z}_{\mathrm{p}}$-class } & \multirow{2}{*}{$\begin{array}{l}\text { Do they } \\
\text { aggree? }\end{array}$} \\
\hline & $\begin{array}{c}\mathrm{L} \\
\text { (long) } \\
\end{array}$ & $\begin{array}{c}\text { I } \\
\text { (intermed.) } \\
\end{array}$ & $\begin{array}{c}\mathrm{S} \\
\text { (short) }\end{array}$ & S & $\mathrm{U}$ & $\mathrm{I} / \mathrm{L}$ & $\mathrm{S} / \mathrm{I}$ & & & \\
\hline $\bar{~} 1$ & 26 & 26 & 19 & 3 & $\bar{~} 4$ & 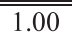 & 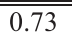 & $\overline{E \text { E(II) }}$ & $\bar{~} \overline{Z \text { Z(I) }}$ & no \\
\hline 2 & 45 & 36 & 17 & 2 & 2 & 0.80 & 0.47 & E(III) & Z(III) & yes \\
\hline 3 & 31 & 27 & 23 & 3 & 3 & 0.87 & 0.85 & $\mathrm{E}(\mathrm{II})$ & Z(II) & yes \\
\hline . & 38 & 29 & 18 & 3 & 2 & 0.76 & 0.62 & $\mathrm{E}(\mathrm{IV})$ & Z(III) & no \\
\hline
\end{tabular}

the classical Zingg case $p=2 / 3$ is assumed). Table 3 shows a sample of our table in which all required data can be recorded.

Instead of assuming a single value for $p$ we let $p$ vary between 0 and 1 and for each value we determined the $Z_{p}$-class. Also, for each value of $p$ we determined the agreement $a(p)$ between $Z_{p}$ - and E-classification: this value is between 0 and 1 and expresses the ratio of pebbles where $Z_{p}$ - and E-classes agreed, i.e. if the pebble was classified in $E(i)$ it was simultaneously classified into $Z_{p}(i)$. The function $a(p)$, drawn in the unit square, we call the $Z / E$ agreement diagram. Figure 6 presents such a Z/E agreement diagram. The diagram has one definite
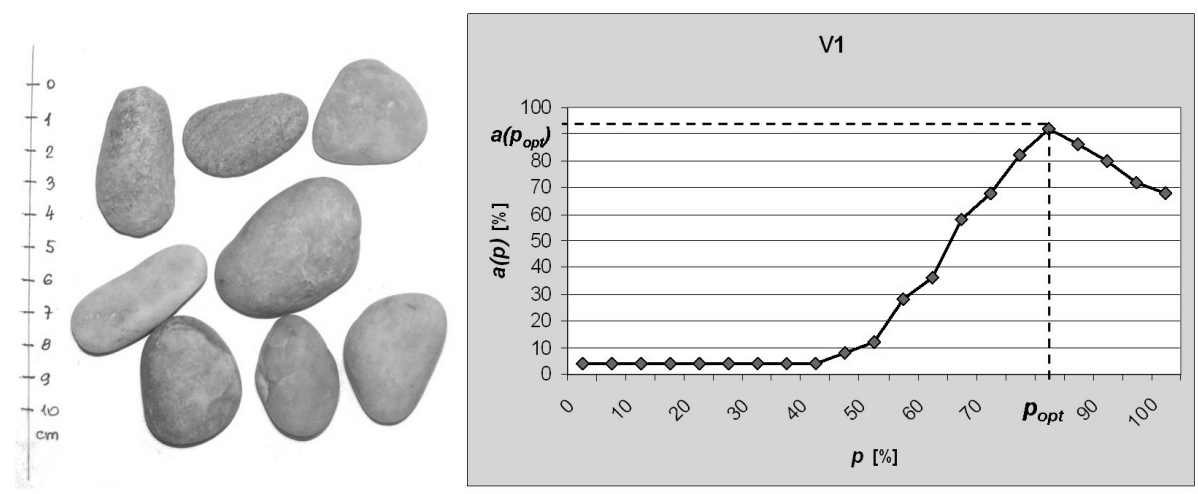

Fig. 6

Some typical pebbles and Z/E aggreement diagram for pebble sample V1. Horizontal axis: parameter $p$ of the generalized Zingg classification (cf. equations (1)-(4)). Vertical axis: agreement a(p) between $\mathrm{Z}_{\mathrm{p}}$ - and E-classification. The diagram has one definite peak at $\mathrm{p}_{\mathrm{opt}}$. The value $\mathrm{a}\left(\mathrm{p}_{\mathrm{opt}}\right)$ is the best match between $Z_{\mathrm{p}}$ - and E-classes 
peak, the p-value associated with this peak we denote by $\mathrm{p}_{\text {opt }}$. The value $\mathrm{a}\left(\mathrm{p}_{\text {opt }}\right)$ is the best match between $Z_{p}$ - and E-classes.

Table 4 summarizes our experimental results of the 20 samples.

We found that for all locations and all samples the Z/E diagram has one (and only one) characteristic peak, in the range $0.55<\mathrm{p}_{\text {opt }}<0.89$. The values $\mathrm{p}_{\mathrm{opt}}$ are listed in a separate column of Table 4 . The value $a\left(\mathrm{p}_{\text {opt }}\right)$, i.e. the best match between $Z_{p}$ - and E-classes, varies between $0.56-0.96$ and is also listed separately, as well as the percentages in all classes at $p=p_{\text {opt }}$.

The definite, single peak in the $\mathrm{Z} / \mathrm{E}$ curves suggests that there exists an optimal Zingg parameter for each sample at which Z-classification works most efficiently. For well-rounded, worn stones the optimal Zingg parameter is higher $(\sim 0.8)$, for more rugged stones it is smaller $(\sim 0.6)$. This shows that the optimal Zingg parameter is not universal; however, Zingg's original guess of $p=0.67$ was a very good one. It also shows that one can estimate the value of $\mathrm{p}_{\text {opt }}$ pretty accurately based on the pebble's shape. The shape of the $\mathrm{Z} / \mathrm{E}$ agreement diagrams is very characteristic for the sample. In the case of well-rounded, worn stones (samples 1-11) the curve starts low and has a long monotonic slope to the peak. In case of rugged shapes (samples 12-20) the curve starts high and has only a minor slope before the peak.

Based on the values a $\left(\mathrm{p}_{\text {opt }}\right)$, we can see that data on Z-classes can be extracted from E-classification with fair ( 10-20\%) accuracy. In our experiments we only used simplified E-classes since our goal was to compare $\mathrm{E}$ - and $\mathrm{Z}_{\mathrm{p}}$-classes. Simplified E-classification contains the same information as the Zingg classes; however, the 'full' equilibrium classification admits more subtle distinction of shapes which cannot be captured by Z-classification. As an example we mention that the Zingg system cannot distinguish between tetrahedral and cubic shapes, while equilibrium classes $\{4,4\}$ and $\{6,8\}$ give clear descriptions of this shapes.

Statistical results also show that there is a significant difference between different geologic locations; this implies that different locations can be distinguished based on equilibrium classification. The distribution of pebbles in the different E-classes can indicate a special abrasion process, depositional environment and/or pebble lithology. For example, collecting pebble samples of identical rock types can help to distinguish between the abrasion of wave-current on a beach, where pebbles are dominantly sliding on each other, and the abrasion in a riverbed, where the case of rolling is more frequent. Analysis of pebble shape can also characterize the environment, as it can separate sandy beaches from gravelly beaches, high-wave energy beaches form low-wave energy ones (Dobkins and Folk 1970). Finally, equilibrium classification also can indicate a special pebble lithology (e.g. schist pebbles are dominantly flat because of the lamellar strucure of this rock type; see samples 1-3).

In this paper we presented pebble samples from various geologic locations to show the applicability of our new system; however, our future project is a 
14 T. Szabó, G. Domokos

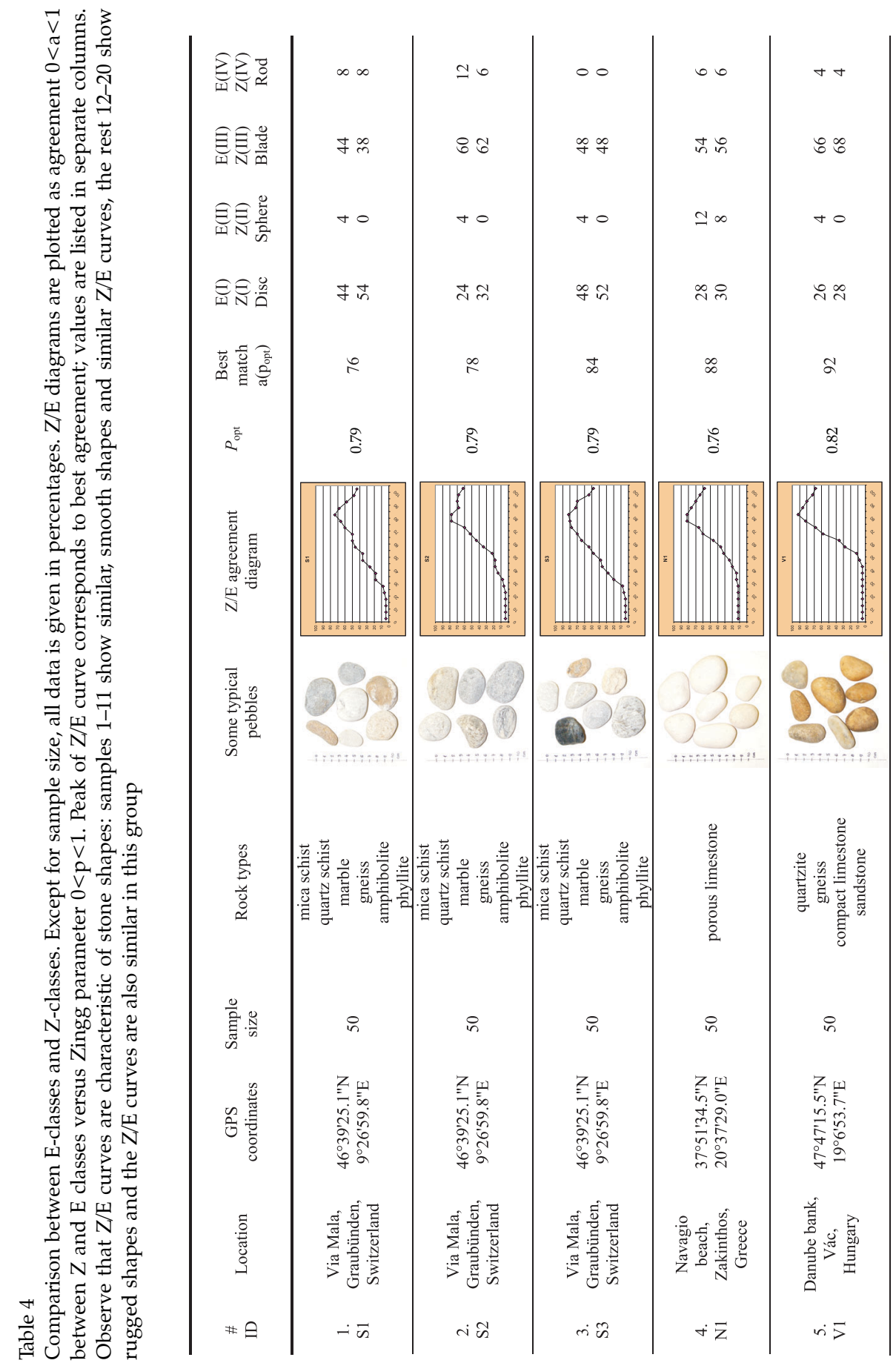

Central European Geology 53, 2010 
A new classification system for pebble and crystal shapes based on static equilibrium points 15

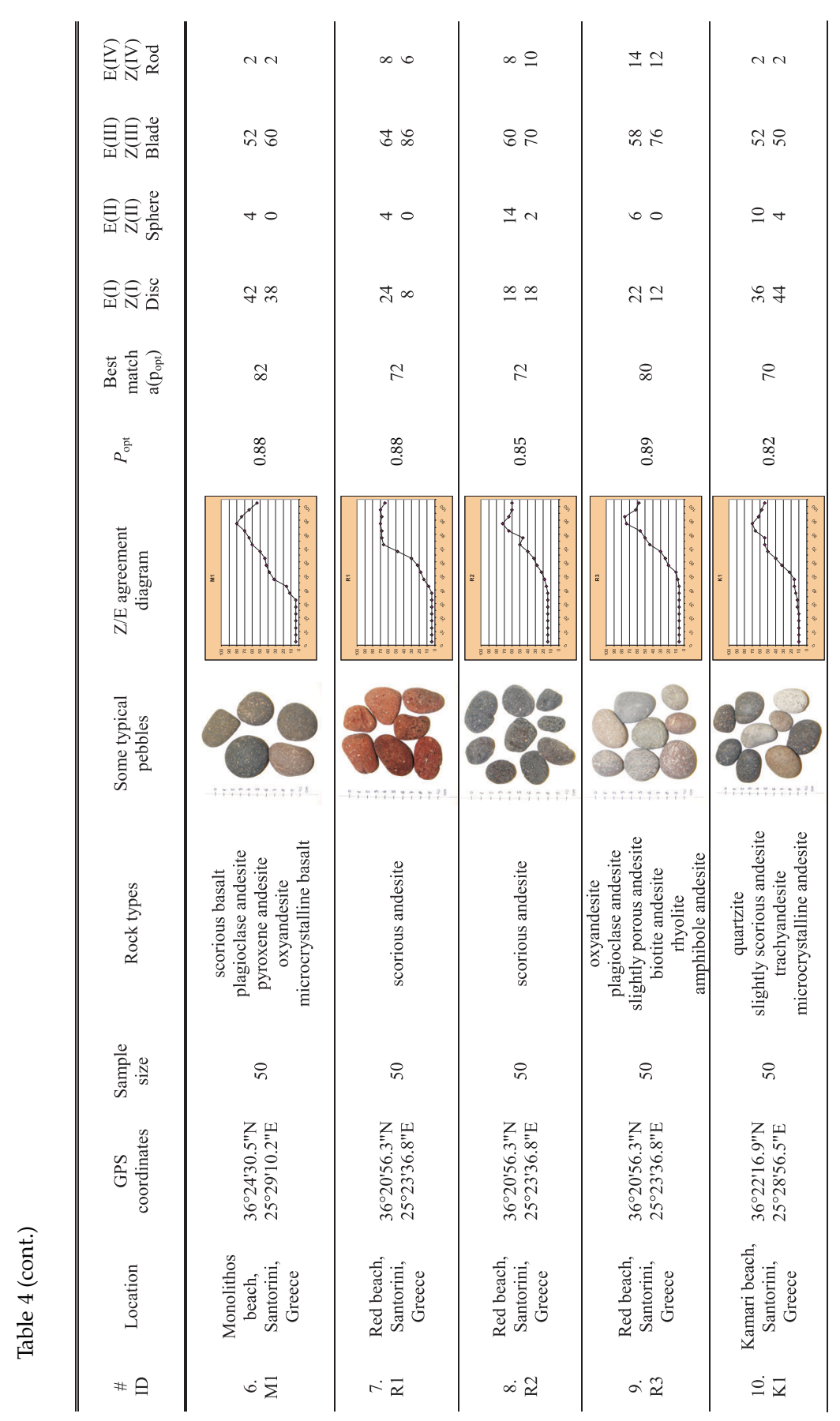

Central European Geology 53, 2010 
16 T. Szabó, G. Domokos

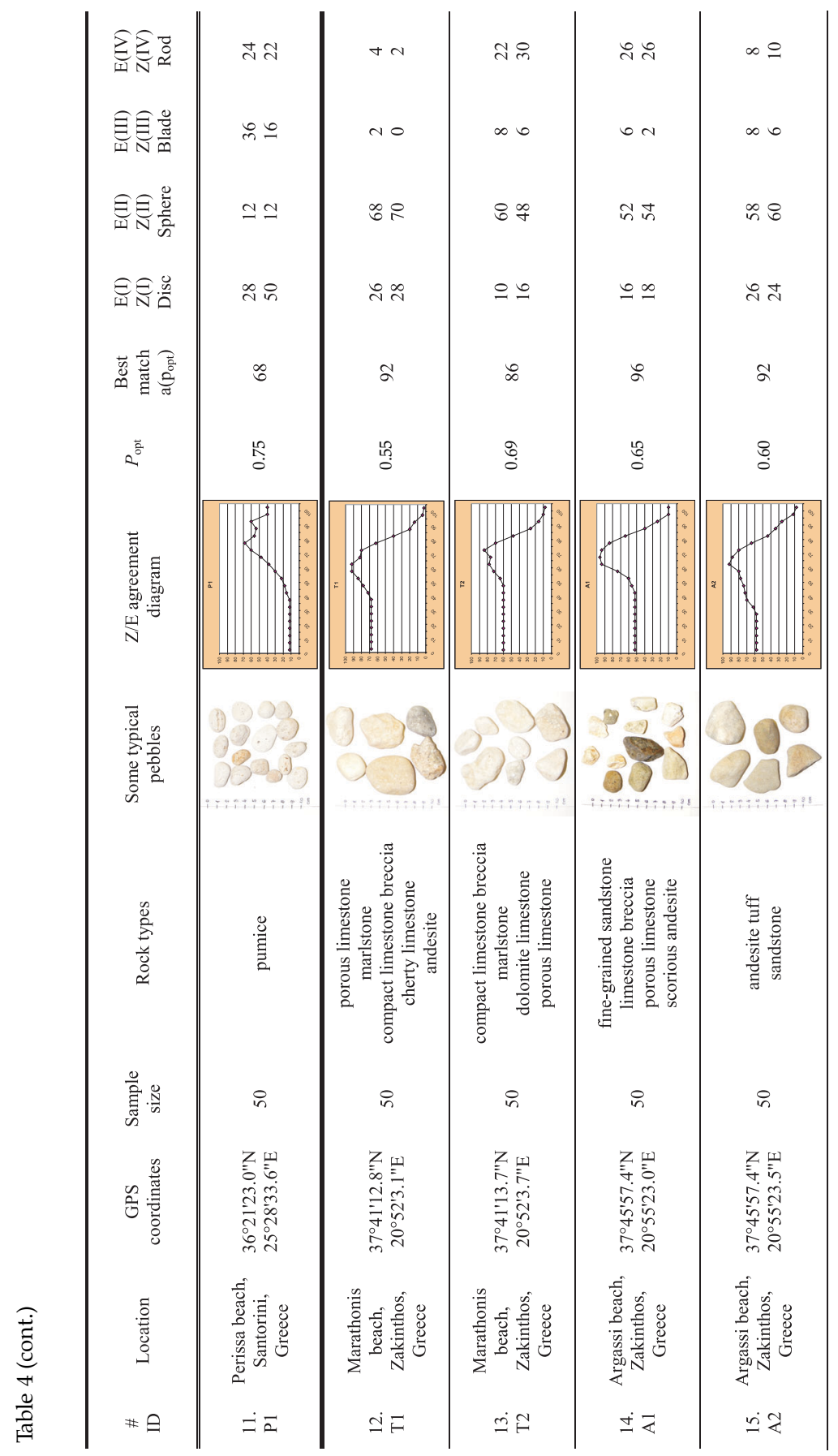

Central European Geology 53, 2010 
A new classification system for pebble and crystal shapes based on static equilibrium points 17

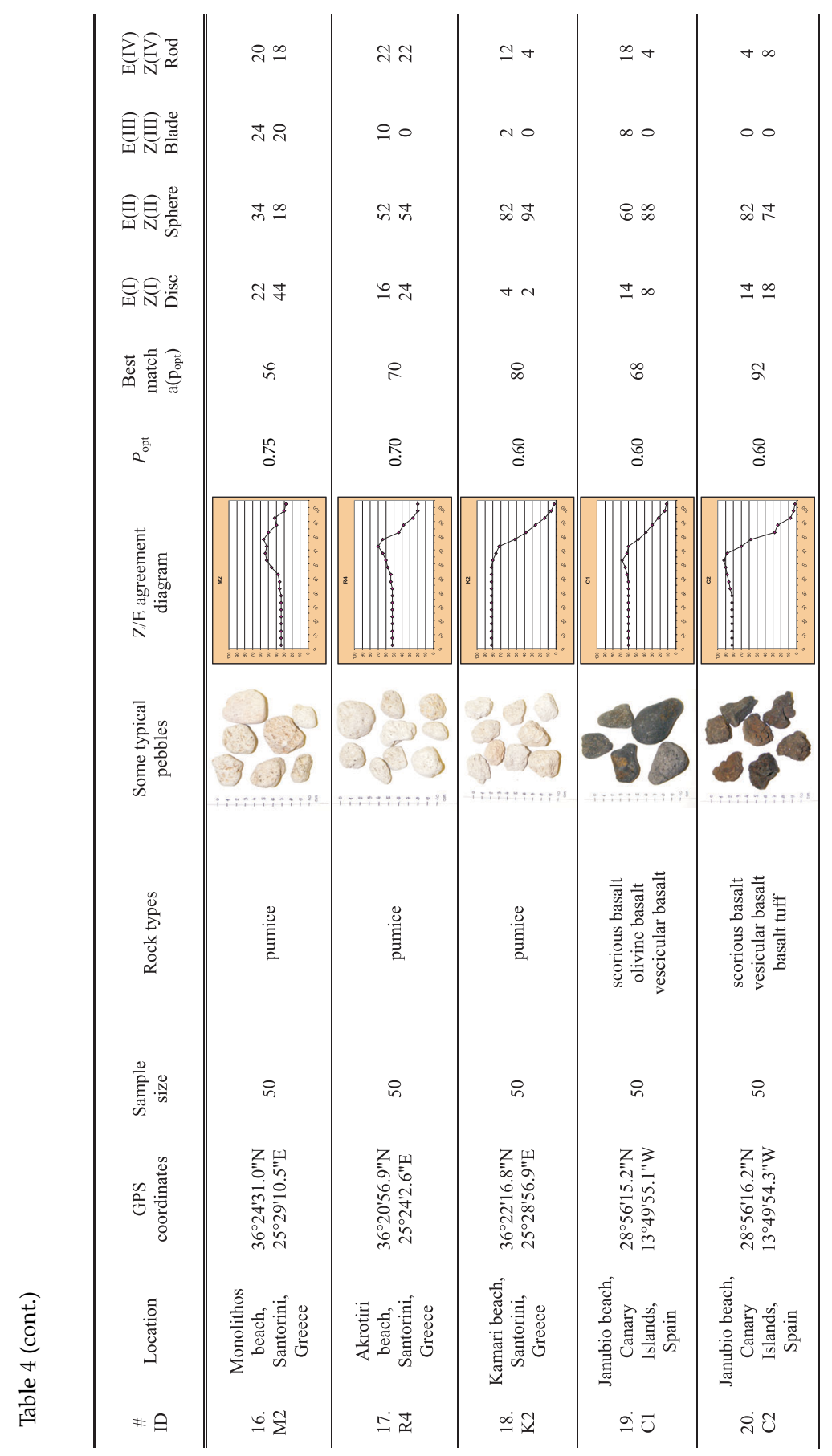

Central European Geology 53, 2010 
purposeful application of the new system, based on geologically interesting pebble samples.

\section{Conclusions}

In our paper we proposed a new classification scheme for pebble shapes, based on the number and type of static equilibrium points. Although our system is more elaborate in the sense that it includes - in theory - infinitely many classes, in practice only half a dozen play an important role. We introduced a simplified version with just four E-classes which is a rapid and adequate tool in most cases. The new system appears to be a natural choice in the sense that the integer number of static equilibria is encoded in the pebble's shape. There is no need to involve any arbitrary constants or directions in the classification system; the only task is to count static equilibrium points of the surface.

We observed that flat shapes always belong to class $\mathrm{E}(\mathrm{I})$, elongated shapes to $\mathrm{E}(\mathrm{IV})$ and by similar argument flat and elongated shapes with small axis ratios $\mathrm{b} / \mathrm{a}, \mathrm{c} / \mathrm{b}$ always fall into class $\mathrm{E}(\mathrm{III})$. This indicates that the number and type of equilibria constrains important shape informations although it does not completely determine the geometric shape.

We compared Zingg classification to E-classification. We found that, depending on the choice of the Zingg-parameter $\mathrm{p}$ a $80-90 \%$ agreement exists, i.e. all the information contained in Zingg classification can be extracted from the simplified equilibrium classification. However, if the "full" counting process is applied, the new system is more sophisticated and is capable of storing information on special (e.g. polyhedral) shapes which cannot be extracted from the Zingg system. In particular, in the case of crystal shapes the new system promises to be useful: equilibrium class is a clear indicator of crystal habit.

Our approach appears to be a natural one and possibly in combination with other methods may help to identify statistical shape features not discovered so far, which may add new information about the wear process and the depositional environment of pebbles.

\section{Acknowledgements}

The authors are grateful to the staff of the Department of Mineralogy, Eötvös Loránd University, for providing access to their collection of minerals. We are most indebted to Ákos Török for his kind help, in particular with identifying rock types. The support of OTKA grant 72146 is appreciated.

\section{References}

Arnold, V.I. 1998: Ordinary differential equations. - 10th printing, MIT Press, Cambridge. Aschenbrenner, B.C. 1956: A new method of expressing particle sphericity. - Journal of Sedimentary Petrology, 26, pp. 15-31. 
Benn, D.I., C.K. Ballantyne 1992: Pebble shape (and size!) - discussion. - Journal of Sedimentary Petrology, 62, pp. 1147-1150.

Blott, S.J., K. Pye 2008: Particle shape: a review and new methods of characterization and classification. - Sedimentology, 55, pp. 31-63.

Corey, A.T. 1949: Influence of shape on fall velocity of sand grains. - unpublished MSc Thesis, Colorado A\&M College.

Dobkins, J.E., R.L. Folk 1970: Shape development on Tahiti-Nui. - Journal of Sedimentary Petrology, 40, pp. 1167-1203.

Domokos, G., A. Sipos, T. Szabó, P. Várkonyi 2010: Pebbles, shapes, and equilibria. - Mathematical Geosciences, 42, pp. 29-47.

Graham, D.J., N.G. Midgley 2000: Graphical representation of particle shape using triangular diagrams: an Excel spreadsheat method. - Earth Surface Processes and Landforms, 25, pp. 1473-1477.

Hahn, T. (Ed.) 2002: International Tables for Crystallography, Volume A: Space Group Symmetry 5th edition, Dordrecht: Kluwer Academic Publishers.

Illenberger, W.K. 1991: Pebble shape (and size!). - Journal of Sedimentary Petrology, 61, pp. 756-767.

Illenberger, W.K. 1992a: Pebble shape (and size!) - reply. - Journal of Sedimentary Petrology, 62, pp. 538-540.

Illenberger, W.K. 1992b: Pebble shape (and size!) - reply. - Journal of Sedimentary Petrology, 62, pp. 1151-1155.

Krumbein, W.C. 1941: Measurement and geologic significance of shape and roundness of sedimentary particles. - Journal of Sedimentary Petrology, 11, pp. 64-72.

Mock, A., D.A. Jerram 2005: Crystal Size Distributions (CSD) in three dimensions: Insights from the 3D reconstruction of a highly porphyritic rhyolite. - Journal of Petrology, 46, pp. 1525-1541.

Oakey, R.J., M. Green, P.A. Carling, M.W.E. Lee, D.A. Sear, J. Warburton 2005: Grain-shape analysis a new method for determining representative particle shapes for populations of natural grains. - Journal of Sedimentary Research, 75, pp. 1065-1073.

Smalley, I.J. 1967: The presentation of subjective shape and roundness data. - Sedimentology, 8, pp. 35-38.

Sneed, E., R.L. Folk 1958: Pebbles in the lower Colorado River, Texas, a study in particle morphogenesis. - Journal of Geology, 66, pp. 114-150.

Várkonyi, P., G. Domokos 2006: Static equilibria of rigid bodies: dice, pebbles and the Poincaré-Hopf Theorem. - Journal of Nonlinear Science, 16, pp. 255-281.

Wentworth, C.K. 1922: The shapes of beach pebbles. - US Geological Survey Professional Paper, 131C, pp. 75-83.

Woronow, A. 1992: Pebble shape (and size!) - discussion. - Journal of Sedimentary Petrology, 62, pp. 536-537.

Zingg, T. 1935: Beitrag zur Schotteranalyse. - Schweizeriscke Mineralogische und Petrologische Mitteilungen, 15, pp. 39-140. 